\title{
Correction to: Outer 1-Planar Graphs
}

\section{Christopher Auer $^{1}$. Christian Bachmaier ${ }^{1}$. Franz J. Brandenburg ${ }^{1}$. Andreas Gleißner ${ }^{1} \cdot$ Kathrin Hanauer ${ }^{1}$. Daniel Neuwirth ${ }^{1}$. Josef Reislhuber ${ }^{1}$}

Published online: 26 September 2021

๑) Springer Science+Business Media, LLC, part of Springer Nature 2021

\section{Correction to: Algorithmica (2016) 74:1293-1320 https://doi.org/10.1007/s00453-015-0002-1}

In Theorem 8 of our article "Outer-1-Planar Graphs" (Algorithmica 74(4), pp. 1293$1320,[1])$, we erroneously claim that every outer 1-planar graph admits a planar visibility representation in $\mathcal{O}(n \log n)$ area. This statement has recently been disproved by Biedl (GD 2020, LNCS 12590, pp. 526-528, [2]), who showed that there are outer 1-planar graphs with an area requirement of $\Omega\left(n^{2}\right)$ in any planar polyline drawing. The other results of our article remain unaffected.

The original article can be found online at https://doi.org/10.1007/s00453-015-0002-1.

$凶$ Christian Bachmaier

bachmaier@fim.uni-passau.de

Christopher Auer

auerc@fim.uni-passau.de

Franz J. Brandenburg

brandenb@fim.uni-passau.de

Andreas Gleißner

gleissner@fim.uni-passau.de

Kathrin Hanauer

hanauer@fim.uni-passau.de

Daniel Neuwirth

neuwirth@fim.uni-passau.de

Josef Reislhuber

reislhuber@fim.uni-passau.de

1 University of Passau, 94030 Passau, Germany 


\section{References}

1. Auer, C., Bachmaier, C., Brandenburg, F.J., Gleißner, A., Hanauer, K., Neuwirth, D., Reislhuber, J.: Outer 1-planar graphs. Algorithmica 74(4), 1293-1320 (2016). https://doi.org/10.1007/s00453-0150002-1

2. Biedl, T.: Drawing outer-1-planar graphs revisited. In: D. Auber, P. Valtr (eds.) Graph Drawing and Network Visualization-28th International Symposium, GD 2020, Vancouver, BC, Canada, 16-18 Sept 2020, Proceedings, Lecture Notes in Computer Science, vol. 12590, pp. 526-528. Springer (2020). https://doi.org/10.1007/978-3-030-68766-3

Publisher's Note Springer Nature remains neutral with regard to jurisdictional claims in published maps and institutional affiliations. 